\title{
Re: A prospective, randomized trial comparing thulium vapoenucleation with holmium laser enucleation of the prostate for the treatment of symptomatic benign prostatic obstruction: perioperative safety and efficacy
}

\author{
Prashant Mulawkar ${ }^{1}$ (D)
}

Received: 23 November 2017 / Accepted: 25 November 2017 / Published online: 4 December 2017

(c) Springer-Verlag GmbH Germany, part of Springer Nature 2017

\section{Dear Editor,}

I read the above-mentioned article with interest. The authors have compared Thulium vapoenucleation (ThuVEP) with Holmium laser enucleation (HOLEP) and found that both the procedures are safe and effective for treatment of symptomatic BPO giving equivalent and satisfactory immediate improvement in urination with low perioperative morbidity. The occurrence of acute post-operative urinary retention (AUR) was higher after HOLEP than ThuVEP (15.2 versus $2.1 \% p<0.022$ )

The laser machines and the power settings that are used in this study are totally different. ThuVEP was done using vela XL at 90 Watt and HOLEP was done using Auriga XL at $39.6 \mathrm{~W}$. This is a comparison of unequals. Moreover, the surgeon performing ThuVEP was more experienced than surgeon performing HOLEP. AJG had performed more than 500 ThuVEP, whereas CN had performed more than 200 HOLEP procedures. This experience is reflected in operative time, enucleation time, enucleation efficiency, and operation efficiency. What is surprising that the weight of adenoma removed is more in ThuVEP as compared to HOLEP; still, it takes less time. Second, one Clavien 3a complication of incomplete morcellation requiring removal of enucleated tissue in local anesthesia and one Clavien $3 \mathrm{~b}$ complication of incomplete morcellation requiring secondary morcellation are added to HOLEP group. These are the complications of morcellation and not of HOLEP.

This comment refers to the article available at https://doi. org/10.1007/s00345-017-2071-z.

Prashant Mulawkar

pmulawkar@hotmail.com

1 Tirthankar Superspeciality Hospital, Akola, India
In HOLEP group, one patient was on apixaban and two patients were on dabigatran. These drugs were not stopped perioperatively, whereas in ThuVEP group, three patients were operated after stopping phenprocoumon or rivaroxaban. It would be interesting to know whether the patients presenting with clot retention were from these groups in HOLEP, because these drugs are associated with increased risk of operative bleeding [1].

Finally, we do not find it necessary to keep patients on bladder irrigation after laser enucleation. In fact, we never put in a three way catheter in these patients. Of course, these must be the surgeon's preference.

The $100 \mathrm{~W}$ Holmium laser machine has the frequency of $50 \mathrm{~Hz}$. With this frequency, the enucleation procedure is quite fast and the bleeding is much less. This I think is because of simultaneous coagulation of the bed due to high frequency. The laser fiber must be getting defocused sometimes, thereby causing coagulation. The coagulation depth of holmium is more than thulium. Therefore, increased bleeding-related complications in holmium are difficult to understand at least theoretically.

There is a trial [2] ongoing in Germany comparing the $50 \mathrm{~W}$ versus $100 \mathrm{~W}$ laser machine. The trial was to start in June 2015 and to end in March 2017. 122 patients were to be recruited in this trial. I think that this trial would answer some of the questions regarding laser machines.

\section{References}

1. Ortel TL (2012) Perioperative management of patients on chronic antithrombotic therapy. ASH Educ Progr Book 2012(1):529-535

2. https://www.smartpatients.com/trials/NCT02737241 (trial NCT02737241, Mans 26-11-2015) 